\title{
CARACTERIZAÇÃO MECÂNICA E MICROESTRUTURAL DE CONCRETO GEO POLIMÉRICO SUBMETIDO À FADIGA*
}

Resumo

\author{
Verônica Scarpini Cândido ${ }^{1}$ \\ Alisson Clay Rios da Silva² \\ Fernanda Santos da $\mathrm{Luz}^{3}$ \\ Sergio Neves Monteiro ${ }^{4}$
}

Cimentos provindos de reações álcali-ativadas abrem novas oportunidades para o futuro, como a utilização de polímeros inorgânicos, conhecidos como geopolímeros. Estes materiais são considerados ecologicamente corretos, pois seu processo de fabricação não envolve a emissão de $\mathrm{CO}_{2}$ e é possível utilizar resíduos industriais como matéria prima. Neste trabalho, foi desenvolvido um concreto de cimento geopolimérico (CCG) e sua resistência à fadiga foi comparada com outro concreto correspondente de cimento Portland (CCP). Foram utilizados alguns parâmetros fixos na dosagem de ambos os concretos, como consumo de aglomerantes, relação água/aglomerante e teor de argamassa. O estudo foi realizado por meio de ensaios dinâmicos, avaliando os efeitos de diferentes níveis de tensões, máximas e mínimas, e frequência aplicada. Os resultados de fadiga nas diversas variações realizadas mostraram um melhor desempenho ao comportamento em fadiga do CCG em relação ao CCP. $\mathrm{Na}$ análise microestrutural foi observada uma melhor aderência matriz/agregado no CCG quando comparado com o CCP, o que pode explicar sua maior resistência perante a fadiga.

Palavras-chave: Geopolímero; Concreto geopolimérico; Fadiga.

\section{MECHANICAL AND MICROSTRUCTURAL CHARACTERIZATION OF GEOPOLYMERIC CONCRETE SUBJECTED TO FATIGUE}

\section{Abstract}

Cements from alkali-activated reactions are unleashing opportunities for the future usage of inorganic polymers, known as geopolymers. These materials are eco-friendly, their manufacturing process does not involve $\mathrm{a} \mathrm{CO}_{2}$ emission and it makes possible the use of industrial waste as raw material. In this work, a Geopolymeric Cement Concrete (GCC) was developed through adequate portions of geopolymer components. Its characteristics were compared with Portland Cement Concrete (PCC), through the establishment of some parameters of design such as consumption of binders, water/aggregates ratio and cement content. The mechanical performance of these concretes was evaluated with emphasis on the fatigue behavior. The results showed a better performance of the fatigue behavior of CCG in comparison to PCC in several variations. Better matrix/aggregate adhesion in the CCG in comparison to CCP was observed in the microstructural analysis, which may explain its greater resistance to fatigue.

Keywords: Geopolymer; Concrete geopolymeric; Fatigue.

1 Bacharel em Ciências Biológicas, D.C, Professora adjunta, Faculdade de Engenharia de Materiais da Universidade Federal do Pará (UFPA), Ananindeua, PA, Brasil. scarpini@ufpa.br

2 Bacharel em Química Industrial, D.C, Professor adjunto, Faculdade de Engenharia de Materiais da Universidade Federal do Pará (UFPA), Ananindeua, PA, Brasil. alissonrios@ufpa.br.

3 Engenheira de Materiais, M.C, Doutoranda em Ciência dos Materiais, Seção de Engenharia Mecânica e de Materiais, Instituto Militar de Engenharia (IME), Rio de Janeiro, RJ, Brasil. fsl.santos@gmail.com

4 Engenheiro Metalurgista, Ph.D. Professor Titular, Seção de Engenharia Mecânica e de Materiais, Instituto Militar de Engenharia (IME), Rio de Janeiro, RJ, Brasil. snevesmonteiro@gmail.com 


\section{INTRODUÇÃO}

O concreto de cimento geopolimérico tem sido objetivo de estudo de diversos pesquisadores nas últimas décadas. Esse material associa elevada resistência e baixo custo de produção tornando-se assim uma alternativa viável para aplicação em diversos setores industriais como o da construção civil, petroquímica, nuclear, aeronáutica, naval e automobilística [1].

No concreto geopolimérico, o geopolímero é utilizado como aglomerante ao invés do cimento Portland [2,3]. Os geopolímeros, também chamados polissialatos, são polímeros inorgânicos que compõem uma classe de materiais que apresentam características físicas e químicas próprias. Na produção de geopolímeros aluminossilicatos são dissolvidos parcialmente com soluções alcalinas contendo $\mathrm{NaOH}$ ou $\mathrm{KOH}[1,5]$. O resultado é uma matriz amorfa com partículas cristalinas dispersas provindas de material não reativo [3,5]. A razão Al/Si é a principal variável no processo de geopolimerização [4,5].

Os geopolímeros cristalinos resultam de condições hidrotérmicas de cura bem controlada [6-8]. Porém, os materiais que apresentam propriedades de interesse para aplicações industriais, como os cimentos geopoliméricos, são amorfos, podendo ser obtidos até mesmo à temperatura ambiente [5].

No que tange o comportamento mecânico desse tipo de concreto, pode-se afirmar que ele possui um comportamento similar ao do concreto de cimento Portland. Este último apresenta, por exemplo, fissuras de natureza frágil quando submetido à fadiga e pouca, ou nenhuma, deformação plástica associada à fissuração. A ruptura ocorre pela iniciação e propagação de microfissuras, sendo que em geral, a superfície de fratura é perpendicular à direção de tensão aplicada [9].

Segundo Silva [3] mostra em seus estudos que as propriedades de fadiga do concreto de cimento Portland limitam-se à aplicação de cargas a uma tensão constante, entretanto, isto não ocorre no campo. O histórico prático de tensões irá determinar níveis de carregamento com tensão variada, números de ciclos, seqüências de carregamentos e tempos de relaxação diferentes.

De acordo com Palmgren [10] e Miner [11] o dano por fadiga pode ser equacionado por meio de um modelo. Esse modelo assume que a resistência à fadiga não consumida pela repetição de uma dada carga fica disponível para a repetição de outras cargas, podendo ser determinada pela Equação 1.

$$
D^{\prime}=\sum n^{\prime} / N^{\prime}=1
$$

onde D' é uma fração de dano, n' é o número de ciclos aplicado num nível determinado de tensão e N' é o número de ciclos que causará a ruptura por fadiga no mesmo nível de tensão. Assim, esse trabalho tem como objetivo determinar as propriedades mecânicas e a microestrutura de concreto geopolimérico submetido à fadiga e compará-las com as de um concreto correspondente utilizando cimento Portland.

\section{MATERIAIS E MÉTODOS}

Para a síntese do concreto geopolimérico foram utilizados os seguintes materiais: como fonte de $\mathrm{Si}$ e $\mathrm{Al}$, um caulim e como fonte suplementar de $\mathrm{SiO}_{2}$, silicato de sódio. Como fonte suplementar de álcalis, $\mathrm{KOH}$ comercial. $\mathrm{A}$ fonte de $\mathrm{CaO}$ foi a escória granulada de alto-forno (EGAF). 
O cimento Portland, utilizado como referência de comparação, foi o cimento de alta resistência inicial do tipo CP V - ARI - RS, da Holcim.

A areia quartzosa foi lavada e seca em estufa, aproveitando-se todo o material passante na peneira de $4,8 \mathrm{~mm}$. O agregado graúdo foi caracterizado como brita graduação 0 (zero) e lavado durante o peneiramento para eliminar o material passante na peneira \#200.

$\mathrm{O}$ traço do concreto de cimento geopolimérico foi definido da seguinte maneira: 1: 1,849: 1,745:0,42 (459kg de aglomerantes sólidos, $849 \mathrm{~kg}$ de areia, $801 \mathrm{~kg}$ de brita e $195 \mathrm{~L}$ de água). O teor de argamassa foi $62 \%$, a relação agregado miúdo/total de agregados foi 51,5\%p., a relação agregado graúdo/total de agregados foi 47,3\%v. A relação água total/aglomerantes sólidos (a/a') foi 0,42 . O volume de pasta geopolimérica foi $37 \%$ e o volume total de agregado foi $63 \%$ do volume total de concreto.

Todos os corpos-de-prova de $(100 \times 100 \times 400 \mathrm{~mm})$ foram moldados em uma única camada e vibrados em mesa vibratória por 15 segundos. Após a moldagem, os corpos-de-prova foram cobertos com filme polimérico e mantidos nos moldes por um período de 48 horas.

Os ensaios de resistência à tração na flexão foram determinados segundo a Norma NBR 12142 [12].

Os ensaios de fadiga foram realizados em uma máquina servo-hidráulica da marca MTS (Material Test System) com capacidade máxima de 10.000 kgf e equipado com osciloscópio.

Nos ensaios de fadiga para ambos os concretos, concreto de cimento geopolimérico e concreto de cimento portland, buscou-se determinar o comportamento destes materiais quando submetidos aos seguintes casos:

Tensão máxima constante: para a frequência de $10 \mathrm{~Hz}$, foi determinado o número de ciclos $(\mathrm{N})$ necessário para a ruptura da amostra quando submetida a $(\mathrm{PR})$ constante até a ruptura; (onde PR é o Percentual da Resistência estática do concreto, em flexão, aplicado como tensão máxima (PR) nos ensaios de fadiga). Os valores de PR aplicados para esta condição foram de 0,70, 0,75, 0,80 e 0,85.

Variação de frequência: com o mesmo valor de PR $(0,75)$ foi determinado o número de ciclos $(\mathrm{N})$ necessários para a ruptura do concreto com frequências de $1 \mathrm{~Hz}$ e 5 $\mathrm{Hz}$;

Tensão máxima variável: para a frequência de $10 \mathrm{~Hz}$, foi determinado o número de ciclos $(\mathrm{N})$ necessários para a ruptura da amostra quando submetida a variações nos valores de PR de uma forma crescente e decrescente (ensaio à tensão variável). $O$ modo de carregamento foi realizado da seguinte maneira: (a) variando a tensão de forma crescente; e (b) variando a tensão de forma decrescente. No primeiro caso, foram determinados três níveis de PR que variaram de forma crescente, estes níveis foram de PR1 $=0,70$, PR2 $=0,75$ e PR3 $=0,80$, sendo que foram aplicados para cada um dos três valores, os seguintes números de ciclos: PR1 $=50.000$ ciclos, PR2 $=30.000$ ciclos e PR3 = livre (até ruptura do corpo-de-prova). No segundo caso, em que os valores de PR variaram de maneira decrescente, foram determinados dois níveis de PR que foram, PR1 =0,85 e PR2 =0,80, sendo aplicado um número de 3.000 ciclos para PR1 e um número de ciclos livre (até a ruptura) para PR2.

A análise das superfícies de fratura dos concretos foi realizada através do Microscópio Eletrônico de Varredura (MEV) modelo JSM 5800 - LV da marca Jeol, equipado com EDS (Energy Dispersive Spectroscopy), da marca Noran. O filamento utilizado foi o de tungstênio e tensão entre 20 e $25 \mathrm{kV}$. 
As amostras para a análise em MEV foram extraídas das superfícies de fratura dos corpos-de-prova após o rompimento dos mesmos nos ensaios mecânicos. Foi realizada a deposição de ouro sobre todas as amostras para evitar o carregamento eletrostático.

\section{RESULTADOS E DISCUSSÃO}

O resultado médio de resistência a tração na flexão encontrado para o Concreto de Cimento Portland (CCP) foi de 5,4 MPa e serviu de base para os ensaios de fadiga. No caso do Concreto de Cimento Geopolimérico (CCG), o resultado encontrado foi de 5,9 MPa.

Foram analisadas também as deformações sofridas para ambos os concretos quando submetidos à aplicação de carga estática através dos ensaios de tração na flexão. Os resultados mostraram uma deformação final para o CCG mais elevada que o CCP. Estes valores estão apresentados na Figura 1.

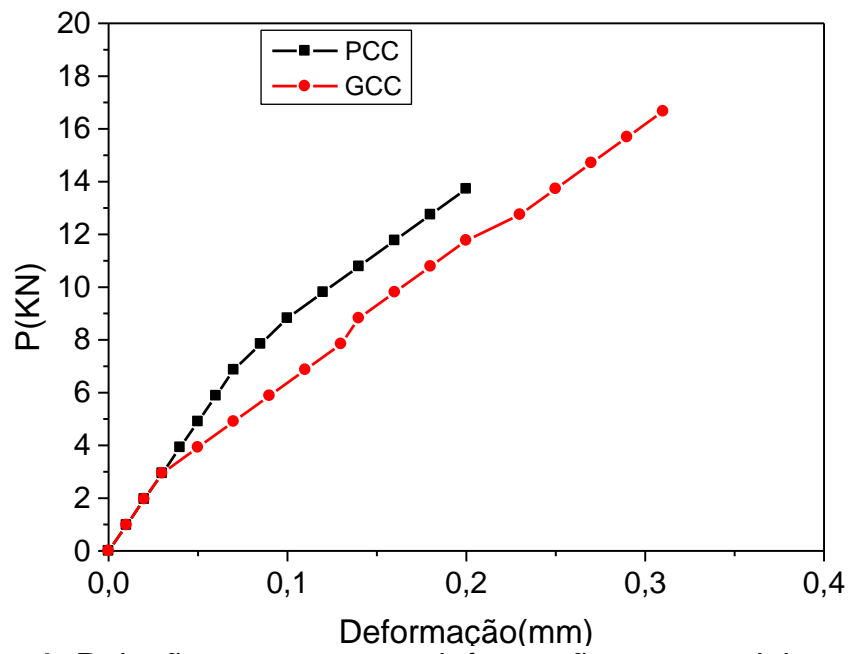

Figura 1. Relação entre carga e deformação para os dois concretos.

A partir da Figura 1 é possível observar que até a carga de $3 \mathrm{kN}$, o CCP e o CCG apresentam o mesmo comportamento de deformação. A partir deste ponto é possível notar uma maior deformação sofrida pelo CCG para cargas mais elevadas. As deformações do CCG atingiram valores da ordem de $0,3 \mathrm{~mm}$, enquanto que 0 CCP os valores encontrados foram de $0,2 \mathrm{~mm}$.

Em relação à carga de ruptura, foi observado que o CCG rompeu somente após aplicação de $17 \mathrm{kN}$, enquanto que o CCP rompeu com $14 \mathrm{kN}$. Este comportamento pode indicar que o CCG é um material mais resistente e mais durável se aplicado em pavimentação.

Os resultados encontrados a partir dos ensaios com diversos valores de PR mostraram que com o aumento da tensão máxima aplicada, a resistência à fadiga de ambos os concretos diminui. A Figura 2 mostra a curva da relação entre tensões versus número de ciclos, para os dois tipos de concreto em estudo. 


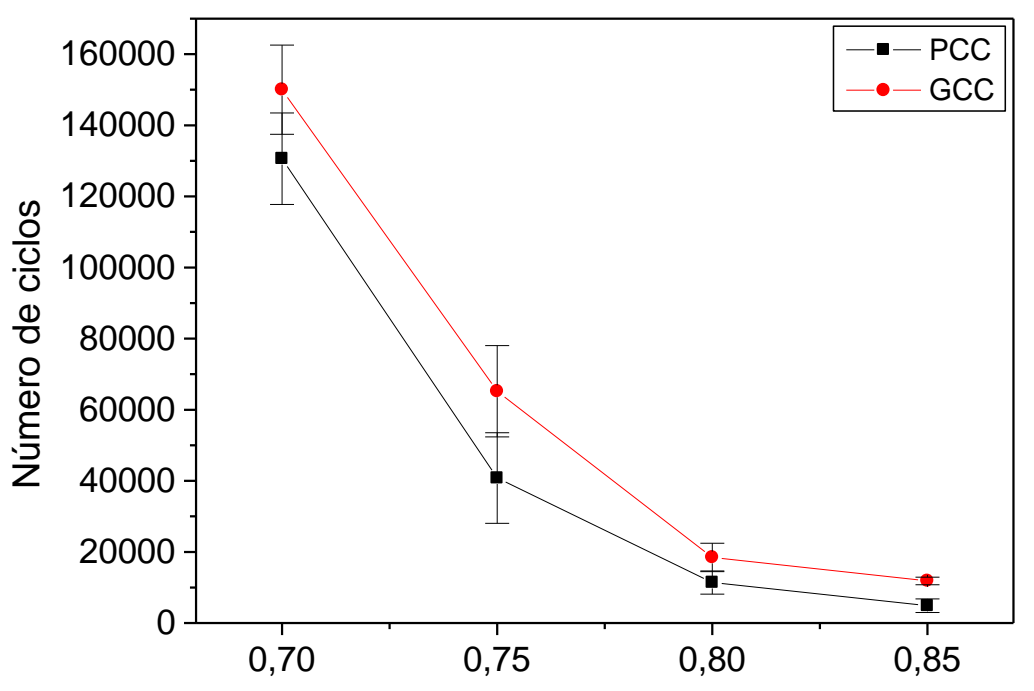

Relacão entre tensões

Figura 2. Variação do Número de ciclos de fadiga com a Relação entre as tensões aplicadas, para o GCC e PCC.

Em contra partida, para níveis de tensão mais baixos, o número de ciclos suportado por cada tipo de concreto aumenta. Estes resultados corroboram com os de outros autores [13], que realizaram ensaios de fadiga em concreto Portland através de três níveis de PR: $0,65,0,75$ e 0,85 . Os resultados obtidos mostraram que a resistência à fadiga do concreto é diferente para os diversos níveis de tensão aplicados, verificando que o número de ciclos sob fadiga para $P R=0,65$ foi superior aos encontrados para as outras relações.

É possível notar que no caso de PR de 0,70 , a diferença média do CCG em relação ao CCP, para as mesmas condições, apresentou valores próximos, considerando a barra de erro. Da mesma forma, para relações entre tensões de 0,85, o CCG exibiu valores similares aos valores encontrados no CCP. Isso pode indicar um comportamento favorável do CCG, quando submetido ao carregamento em fadiga, para altos e baixos níveis de PR aplicada.

Para o estudo da influência da frequência na fadiga dos concretos, foram realizados ensaios com frequências de $1 \mathrm{~Hz}, 5 \mathrm{~Hz}$ e $10 \mathrm{~Hz}$, adotando-se o mesmo valor para PR de 0,75 . A Figura 3 mostra os resultados encontrados.

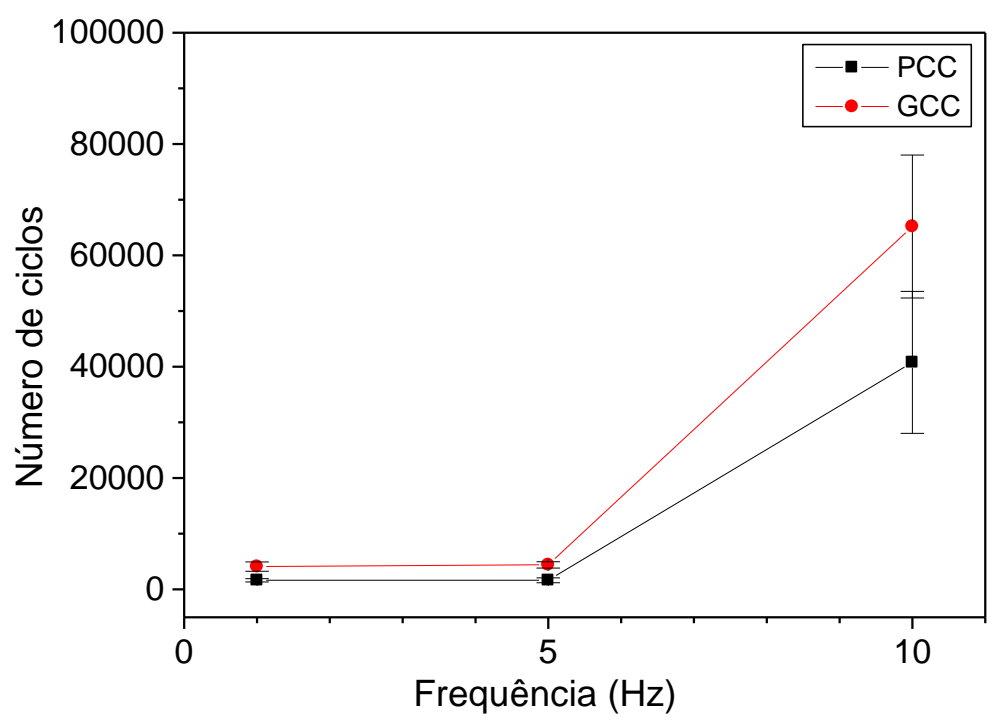

Figura 3. Relação entre a frequência e o número de ciclos alcançados. 
Nota-se que quanto mais elevada for a frequência aplicada, maior o número de ciclos alcançados para o CCG. Outros autores [14] também observaram em seus estudos de fadiga, em CCP, que quanto menor for a frequência aplicada, menor 0 número de ciclos alcançado.

Foi possível concluir, a partir dos resultados, que para a aplicação da frequência de $10 \mathrm{~Hz}$ a vibração do material é muito rápida, o que não permite o alívio completo das tensões dentro do mesmo ciclo de carregamento. Neste caso ocorre um maior efeito de tração e um menor efeito de fadiga. Já para a aplicação de $5 \mathrm{~Hz}$ a vibração é mais lenta, havendo o alívio parcial das tensões dentro de um mesmo ciclo de carregamento. $\mathrm{O}$ efeito neste caso é mais acentuado tanto em fadiga quanto em tração. No menor nível de frequência aplicado, $1 \mathrm{~Hz}$, a vibração é muito lenta, ocorrendo assim um alívio total das tensões dentro de um mesmo ciclo de carregamento. Neste caso há um efeito moderado de tração e fadiga, além de um maior tempo sem carregamento.

Para todos os níveis de frequência aplicados os ganhos de resistência à fadiga para o CCG, foram muito próximos aos alcançados pelo CCP. No caso de pavimentos de concreto, a frequência é um dos principais fatores que influenciam na durabilidade [15]. De acordo com os resultados expostos, o CCG apresenta comportamento favorável à variação de frequências a que os pavimentos estão sujeitos em campo.

Os ensaios com tensão variável foram realizados através da variação de PR de forma crescente e decrescente. Os resultados mostram que quando a tensão é aplicada de forma decrescente, a hipótese para a determinação do dano acumulado pode não ser segura, como mostra a Figura 4.

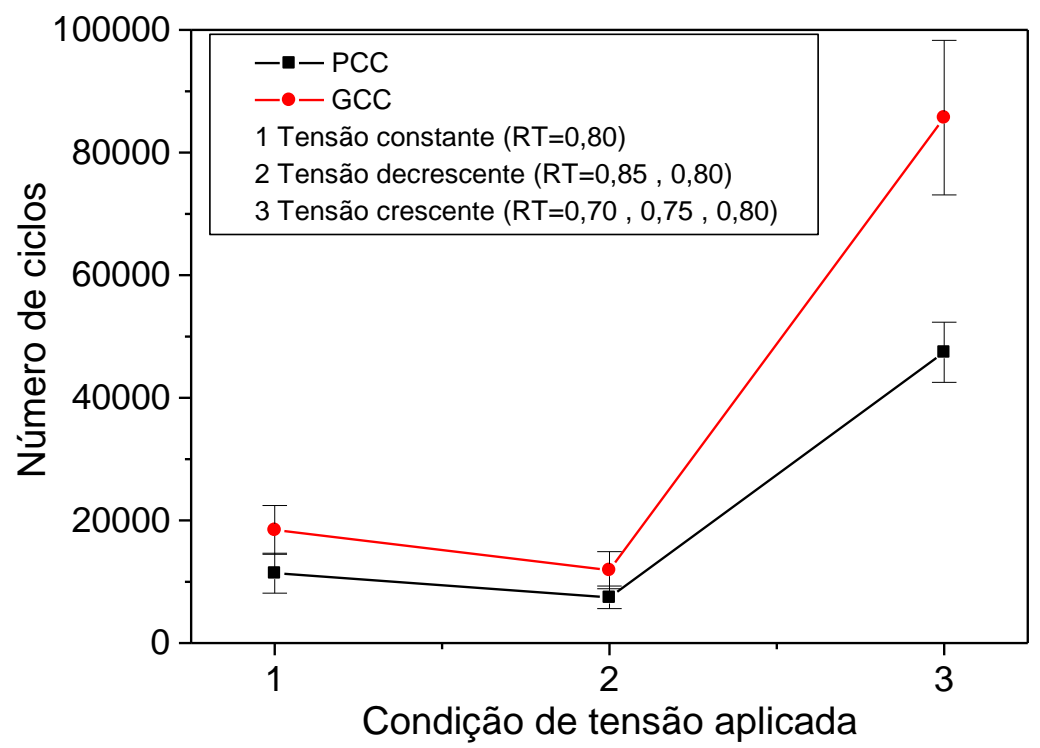

Figura 4. Influência das condições de aplicações de tensão para os dois concretos estudados.

É possível observar que existe pouca influência da variação de tensões, quando esta é aplicada de forma crescente, no comportamento dos concretos sob fadiga. Estas observações também foram feitas por diversos autores [16,17], através da aplicação de dois estágios de tensão. Aplicando níveis de tensão que iam de alto para baixo e níveis de baixo para alto, os autores puderam concluir que a tensão quando aplicada de baixa para alta, a hipótese de Palmgren [10] e Miner [11] seria correta; entretanto isto não era observado variando a tensão de alta para baixa, ou seja, a hipótese nestas condições conduzia a erro nos resultados. 
É possível observar ainda que o número de ciclos obtidos foi menor do que àqueles encontrados nos ensaios com PR constante $(P R=0,80)$, e inferiores também àqueles obtidos para tensão variável de forma crescente. Isto indica a maior influência da variação de tensões, quando aplicada de forma decrescente, no comportamento do concreto sob fadiga. Estes resultados concordam com aqueles encontrados por Maggi [18], onde a autora observa que a menor resistência à fadiga é encontrada em tensões decrescentes.

Quanto ao desempenho dos concretos nas variações de tensões, observa-se, novamente, um melhor desempenho do concreto geopolimérico, quando aplicado tensões crescentes e decrescentes comparado ao concreto Portland.

A micrografia obtida da superfície de fratura dos corpos de prova ensaiados em fadiga está apresentada na Figura 5 (a) e (b).

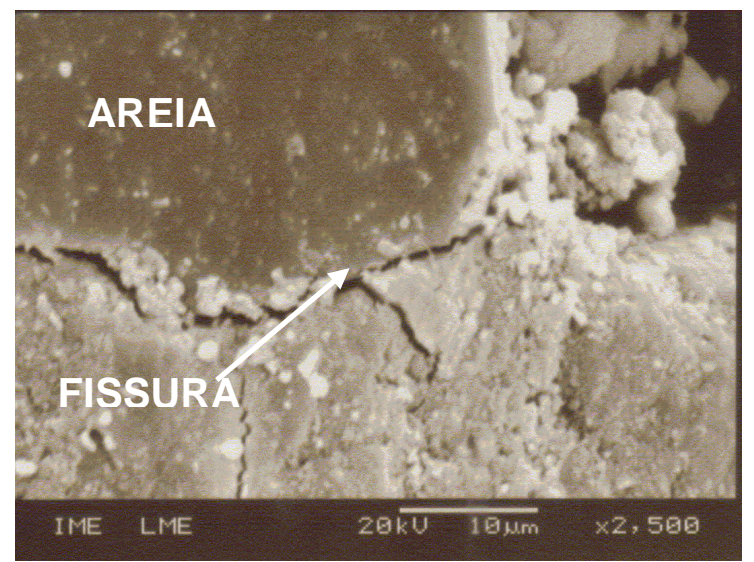

(a)

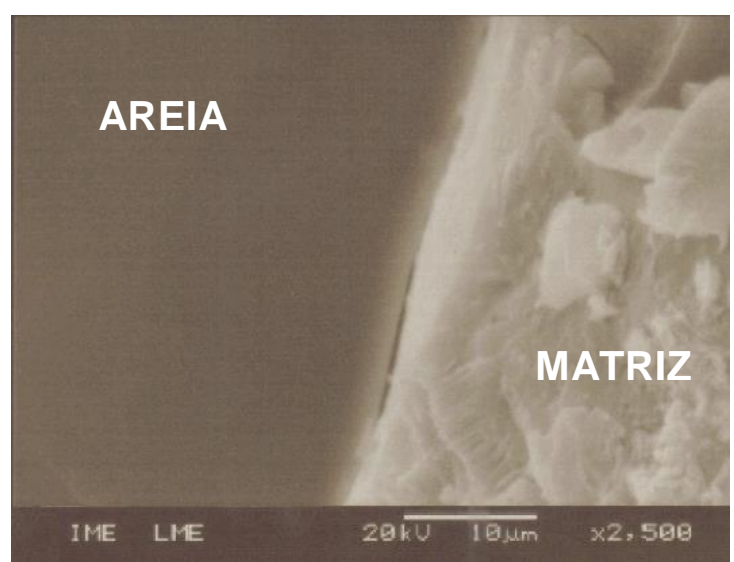

(b)

Figura 5. Micrografias obtidas por MEV de ambos os concretos com PR 0,75 e 10Hz: (a) Região de transição no CCP. (b) Interface matriz agregado no CCG, após o carregamento cíclico.

É possível observar, Figura 5, que a fissura tende a propagar pela interface matriz agregado em ambos os casos. Entretanto, no CCP observa-se uma maior degradação na região interfacial devido a porosidade e a presença de cristais de portlandita, o que limita consideravelmente as propriedades mecânicas do CCP [5]. No caso do CCG, a boa aderência entre a matriz e a areia, após a aplicação do carregamento cíclico, Figura 5 (b), pode ser justificada pela inexistência de cristais de portlandita que fragilizam a interface matriz/agregado.

Os cristais de portlandita presentes na região de interface matriz/agregado do CCP podem ser observados na Figura 6.

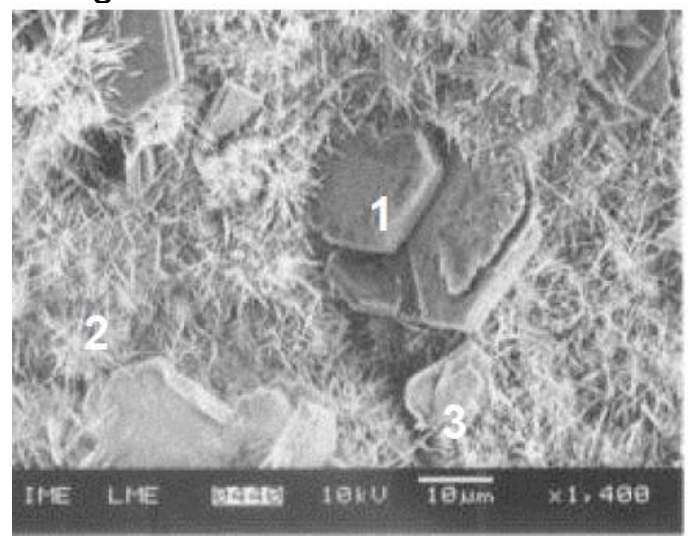

Figura 6. Cristais de Portlandita presentes na região de interface matriz agregado do CCP: (1) cristais de portlandita. 
A portlandita é uma fase secundária do CCP, inexistente no CCG devido as diferentes reações químicas que estão envolvidas no processo de geopolimerização $[19,20]$. Sua ausência no CCG pode explicar a melhor aderência entre a matriz e o agregado [5].

Diante dos resultados e análises apresentados é possível observar que o CCG, submetido a carregamentos cíclicos, apresenta resistência a fadiga igual ou superior em relação ao CCP, dependendo de cada caso. O bom desempenho mecânico do CCG pode ser justificado pela boa aderência agregado/matriz, que não apresenta degradação nesta região devido a inexistência de cristais que fragilizam está interface. Assim, o CCG apresenta potencial de aplicação como pavimento rígido de concreto.

\section{CONCLUSÃO}

- Nos ensaios de flexão é possível notar uma maior deformação sofrida pelo concreto de cimento geopolimérico para maiores cargas estáticas aplicadas.

- Nos ensaios de fadiga com variação da frequência o concreto de cimento geopolimérico (CCG) apresentou um desempenho, em relação a vida útil, $66 \%$ maior que a do concreto de cimento Portland (CCP), devido a sua maior deformação encontrada nos ensaios de módulo de elasticidade e tração na flexão.

- Relações entre tensões de 0,70, a diferença média do CCG em relação ao CCP, para as mesmas condições, apresentou valores superiores. Para relações entre tensões de 0,85 , o CCG exibiu valores mais elevados, quando comparado ao CCP. Isto indica um comportamento favorável sob fadiga do CCG, não só em tensões baixas, como também em tensões mais altas.

- A resistência à fadiga, em testes com a variação de tensões em ambos os tipos de concretos, foi maior do que quando submetido à resistência à fadiga com tensão constante. Isto somente ocorreu quando a tensão variou de baixa para alta (tensão crescente). Para a variação de tensão de um nível mais elevado para um mais reduzido, o número de ciclos obtido foi inferior àquele alcançado com tensão constante.

\section{Agradecimentos}

Quando houver Auxílios ou Bolsas provenientes de órgãos de fomento, os autores deverão fazer referência ao nome da instituição financiadora da pesquisa, ao ano e ao número do processo a que se refere o apoio.

Demais casos são opcionais.

\section{REFERÊNCIAS}

1 Davidovits J. 30 Years of Successes and Failures in Geopolymer Applications. Market Trends and Potential Breakthroughs. Keynote Conference on Geopolymer Conference., Melbourne, Australia, 2002.

2 Rangan BV, Pan Z, Snajyan JG. An investigation of the mechanisms for strength gain or loss pfgeopolymer mortar after exposure to elevated temperature. J. Mater Sci. 2009; 44: 1873-1880.

3 Silva ACR. Comportamento do concreto geopolimérico para pavimento sob carregamento cíclico. 2006. 184p. Dissertação (Mestrado em Ciência dos Materiais) Instituto Militar de Engenharia, 2006. 
4 Duxson P. The Structure and Thermal evolution of Metakaol in Geopolymers. Tese de Doutorado em Filosofia da Engenharia. Universidade de Melbourne. Austrália, Fevereiro, 2006.

5 Silva FJ. Reforço e Fratura em Compósitos de Matriz Álcali-ativada. 269 p. Tese de Doutorado em Ciências dos Materiais - Instituto Militar de Engenharia (IME) - Rio de Janeiro, 2000.

6 Barcosa VFF. Síntese e caracterização de polissialatos. Tese de Doutorado em Ciência dos Materiais, Instituto Militar de Engenharia, 1999.

7 Deventer JSJV, Provis JL. Geopolymerisation kinetics. 1. In situ energy-dispersive Xray diffractometry. Chemical Engineering Science. 2007; 62: 2309-2317.

8 Mackenzie KJD, Barbosa VFF. Synthesis and thermal behavior of potassium sialate geopolymers. Materials Letters. 2003; 57: 1477-1482.

9 Callister Jr WD. Materials Science and Engineering. 7th ed. New York: John Wiley \& Sons, Inc, 2007

10 Palmgren A. Die Lebensdauer von kugellagern VDI. Z. Ver. Deutsch. Ingen. 1924; 68 (14): 339-341.

11 Miner MA. Cumulative Damage in Fatigue. Trans. ASME. 1945; 67: 159-164.

12 Associação Brasileira de Normas Técnicas. ABNT NBR 12142 (MB3483): Concreto Determinação da resistência à tração na flexão em corpos-de-prova prismáticos. Rio de Janeiro. Dezembro. 1991.

$13 \mathrm{OH} \mathrm{BH}$. Cumulative damage theory of concrete under variable-amplitude fatigue loadings. ACI Materials Journals. 1991a; 88: 41-47.

14 Crepaldi APP. Contribuição ao estudo da fadiga do concreto. Anais do $43^{\circ}$ Congresso Brasileiro do Concreto - IBRACON. 2001; 3: 193-208.

15 Aiticin PC. Concreto de Alto Desempenho. 1 ed. São Paulo: Pini, 2000.

16 Cervo TC, Baldo JT, Curti R, Silva C. Estudo sobre medidas de resistência à tração na flexão de concretos para pavimentos com emprego de amostras reduzidas. In: Anais do 46을 Congresso brasileiro do Concreto - IBRACON, Florianópolis, 2004.

17 Zhang $\mathrm{B}$, Wu K. Residual fatigue strength and stiffnes of ordinary concrete under bending. Cement and Concrete Research. 1997; 27(1): 115-126.

18 Maggi PLO. Comportamento de pavimentos de concreto estruturalmente armados sob carregamentos estáticos e repetidos. 205p. Tese de Doutorado em Engenharia Civil Universidade de São Paulo - São Paulo, 2004.

19 Davidivits J. Geopolymers Inorganic Polymeric New Materials. Journal of Thermal Analysis. 1991; 37: 1633-1656.

20 Deventer JSJV, Provis JL. Geopolymerisation kinetics. 1. In situ energy-dispersive Xray diffractometry. Chemical Engineering Science. 2007; 62: 2309-2317. 